\title{
Filosofía de las matemáticas como apoyo al aprendizaje de los números
}

\section{Philosophy of mathematics to support the learning of numbers}

Recibido 25/09/2020

Rafael Rubén Sanic Maguirre

Maestría en Filosofía

Universidad de San Carlos de Guatemala

Ingeniero Industrial

rubensanic@gmail.com

Claudia Esmeralda Marisol Villela Cervantes

Doctora en Educación

Universidad Mariano Gálvez de Guatemala

villelaclaudiaesmeralda@gmail.com

https://orcid.org/0000-0002-8577-4376

Aceptado 18/01/2021

\section{Referencia}

Sanic-Maguirre, R. R. y Villela Cervantes, C. E. (2021). Filosofía de las matemáticas como apoyo al aprendizaje de los números. Revista Docencia Universitaria, 2(1), 62-70.

https://doi.org/10.46954/revistadusac.v2i1.25

\section{Resumen}

El problema que existe en educación media en Guatemala en los últimos tres años es el bajo porcentaje de estudiantes que aprobaron Matemática según las evaluaciones realizadas por el Ministerio de Educación en 2017 el 14.50\% en 2018 14.89\% y 2019 fue 13.56\%. Existen diversas causas por las cuales los estudiantes no aprenden matemática. Se considera efectivo que los docentes utilicen la reflexión filosófica de la matemática como una metodología que motive a los estudiantes a desear aprender matemática. El objetivo del artículo es dar respuesta a las dos preguntas ¿es efectiva la metodología de reflexión filosófica en el aprendizaje de la matemática? ¿Cómo aplicaron la matemática los hombres de ciencia? La metodología que se utilizó en 
el estudio corresponde a la racionalista, empírica y a priori, a través de la investigación documental sobre Descartes, quien, con su creación de geometría analítica, descubrió el sistema de coordenadas, hasta la fecha es utilizada en el posicionamiento por satélite. Arquímedes, con sus inventos geométricos, utilizados en Hidráulica, Newton inventor del cálculo diferencial, matemáticas que le sirvieron para demostrar los principios de la física clásica y finalmente Gauss, quien dedicó su vida al estudio del álgebra y estadística, sus conocimientos estadísticos se aplican en la actualidad en todas las disciplinas en todo el mundo.

\section{Abstract}

The problem that exists in high school education in Guatemala in the last three years is the low percentage of students who passed Mathematics according to the evaluations carried out by the Ministry of Education in $2017,14.50 \%$ in $2018,14.89 \%$, and in 2019 it was $13.56 \%$. Surely there are various reasons why students do not learn mathematics. It is considered effective that teachers use the philosophical reflection of mathematics as a methodology that motivates students to want to learn mathematics. The objective of the article is to answer the two questions: is the methodology of philosophical reflection effective in learning mathematics? How did scientists apply mathematics? The methodology used in the study corresponds to the rationalist, empirical and a priori, through documentary research on Descartes, who, with his creation of analytical geometry, discovered the coordinate system, to date it is used in positioning by satelite. Archimedes, with his geometric inventions, used in Hydraulics, Newton inventor of the differential calculus, mathematics that served to demonstrate the principles of classical physics and finally Gauss, who dedicated his life to the study of algebra and statistics, his statistical knowledge is applied in the present in all disciplines around the world.

\section{Introducción}

En los últimos dos años es bajo el porcentaje de estudiantes que aprobaron Matemática según las evaluaciones realizadas por el Ministerio de Educación en el año 2017 el 14.50 \% aprobaron el examen, para el año 2018 ganaron el examen únicamente el 14.89\% y en el año 2019 aprobaron 13.56\%. Sin embargo, son dos décadas de obtener bajos resultados en las evaluaciones de matemática y se atreve a pensar que en la historia de la educación siempre ha sido así.
Palabras clave:

filosofía, matemática, aprendizaje
Keywords: philosophy, mathematics, learning 
Los resultados deficientes reflejan que a los estudiantes no les gusta la matemática, precisamente porque al parecer no le encuentran utilidad a la aplicación de los números. Para ello se ha experimentado por la experiencia docente de más de 30 años en nivel medio, que a los estudiantes les gusta mucho conocer los aportes y el uso de la matemática de los grandes científicos.

Se reconoce que la filosofía de las matemáticas tiene como objetivo la reflexión y el razonamiento ante los fenómenos de la vida real, y con ello tratan de interpretar y reflexionar el lenguaje, el método, el objeto y la naturaleza, entonces al referirse a la filosofía y matemática son dos ciencias abstractas, que tienen muchos puntos en común como por ejemplo la forma de abordar los problemas y las preguntas de los fenómenos de la vida misma.

El objetivo del artículo es dar respuesta a dos preguntas ¿Es efectiva la metodología de reflexión filosófica en el aprendizaje de la matemática? ¿Cómo aplicaron la matemática los hombres de ciencia? La metodología que se utilizó en el estudio corresponde a la racionalista, empírica y a priori, a través de la investigación documental sobre Descartes, quien, con su creación de geometría analítica, descubrió el sistema de coordenadas, hasta la fecha es utilizada en el posicionamiento por satélite. Arquímedes, con sus inventos geométricos, utilizados en Hidráulica. Newton inventor del cálculo diferencial, matemáticas que le sirvieron para demostrar los principios de la física clásica y finalmente Gauss, quien dedicó su vida al estudio del álgebra y estadística, sus conocimientos estadísticos se aplican en la actualidad en todas las disciplinas del mundo. Indudablemente conocer a profundidad el aporte a la ciencia de los grandes científicos favorecerá el aprendizaje de la matemática a los estudiantes y por lo consiguiente facilitará resolver problemas elementales de la vida diaria.

\section{Materiales y métodos}

El método que se utilizó en la investigación, fue el método deductivo se partió de lo general, se analizó los conocimientos obtenidos por la experiencia de haber atendido cursos de matemática por más de 30 años. Se realizó investigación documental la cual consistió en la revisión del libro ¿es Dios un matemático? de Mario Livio, a sí mismo se investigó en Google Académico diferentes biografías de científicos matemáticos. 


\section{Resultados}

En el informe de resultados generales de la evaluación educativa realizada por el Ministerio de educación de Guatemala, Mineduc (2009), dio a conocer los resultados de la evaluación de matemática para graduandos correspondientes al año 2017 el 14.50 \% aprobaron el examen, para el año 2018 ganaron el examen únicamente el 14.89\% y en el año 2019 aprobaron 13.56\%. Los resultados son los más altos y corresponde a la ciudad capital, en los departamentos los resultados fueron menores hubo departamentos que su porcentaje de alumnos aprobados en matemáticas fueron del cinco por ciento. Después de analizar estos resultados tan deficientes y deplorables. Se llega a la conclusión que, no se cumplieron las metas y objetivos, porque no se usó una buena metodología que favorezca el alcance de competencias en matemática.

La filosofía y la matemática son dos disciplinas abstractas. La filosofía trata de buscar el porqué de las cosas y las matemáticas buscan solución a problemas numéricos; así que la matemática es una materia importante en la filosofía y viceversa, también estas materias tienen en común que las dos utilizan el pensamiento o conocimiento para resolver diferentes problemas.

El conocimiento matemático es la facultad que disponemos para hacer frente a un determinado problema. A través de la reflexión sobre ejercicios y sus soluciones dentro de un contexto social.

Los fundamentos de las matemáticas son el estudio de conceptos matemáticos básicos como números, ecuaciones, figuras geométricas, conjuntos, funciones, etc. Hay otros aspectos interesantes de la matemática que atraen de modo natural a los estudiantes. La dinámica interna del pensamiento matemático, la lógica de su estructura, simple, tersa, sobria, clara, hacen de ella un modelo de reflexión fiable que suscita el consenso de todos interesados en aclarar los misterios del conocimiento humano; han visto en el pensamiento matemático un campo ideal de trabajo donde poner a prueba sus hipótesis y teorías.

¿Son las matemáticas una creación humana o un descubrimiento? Desde la antigüedad hasta el presente, científicos y filósofos se han maravillado que una disciplina tan abstracta pudiera explicar de manera tan perfecta el mundo natural (Livio, 2009). 
La filosofía de las matemáticas es una parte de la filosofía, que trata de interpretar y explicar el lenguaje, el objeto, el método y la naturaleza de las matemáticas. Las matemáticas son básicamente una extensión de la lógica.

¿Por qué? y ¿Para qué? estudiar matemáticas, es lo que con frecuencia se preguntan los estudiantes. ¿Por qué debemos estudiar matemáticas? Debemos tener un pensamiento crítico; como estudiante, como trabajador de una empresa o para dirigir su propia empresa y como conocimiento general. El pensamiento crítico lo obtenemos con conocimientos matemáticos y perfeccionando la razón, ha sido considerado por algunos filósofos como el modelo de la tradición racionalista moderno.

Ruiza, Fernández, y Tamaro, (2004) se hacen las preguntas ¿Para qué hay que estudiar matemáticas? La respuesta la tienen los científicos Descartes, Arquímedes, Newton y Gauss. René Descartes nació en 1596 y falleció en 1650, fue filósofo y matemático francés, considerado el padre del racionalismo y también de la geometría analítica, como lo muestra en su libro "Discurso del método" publicado en 1637 y donde resalta su pensamiento "pienso, luego existo" (en latín cogito, ergo sum). Él mencionó que dudar también es una forma de pensar, se puede dudar de todo menos de la persona que está pensando.

Descartes con su geometría analítica, desarrolló el eje de sistemas de coordenadas que son dos rectas perpendiculares que se cortan en un solo punto llamado origen, se le denominó plano cartesiano, que es la base de la posición satelital hoy en día muy usado en las diversas disciplinas. También se agrega el método racionalista el cual coloca a las matemáticas como una forma de ver las causas que originan el fenómeno.

Arquímedes (ca. 287 a. C. ca. 212 a. C) junto con Isaac Newton y Gauss, están considerados como los tres matemáticos más grandes que han existido en la humanidad. Fueron muchos los inventos de Arquímedes, adquirió fama por sus invenciones mecánicas; su fascinación por la geometría lo hizo inventar máquinas de guerra, útiles que los ejércitos utilizaron para ganar batallas y murió en manos de un soldado que lo asesinó cuando él se encontraba en la arena de una playa haciendo cálculos geométricos. Es muy popular su frase "Dadme un punto de apoyo y moveré el mundo" (Ruiza, Fernández, y Tamaro, 2004). 
Isaac Newton nació en 1642 y falleció en 1727, fue filósofo, físico y matemático inglés que consagró su vida a la investigación científica y a la religión cristiana. Se le reconoce como una de las grandes mentes de la Revolución científica del siglo XVII. Su obra cumbre fue publicada en 1687 "Philosophiae naturalis principia mathematíca", los principios matemáticos de la filosofía natural. El contenido de esta obra se refiere a la física y la astronomía escritos en el lenguaje de la geometría pura. La frase de su autoría "Un hombre puede imaginar cosas que son falsas, pero sólo puede entender cosas que son ciertas". Se le considera el creador del Cálculo diferencial e integral, paralelamente con el matemático y filósofo alemán Leibniz. Esta matemática sirvió de base para demostrar sus inventos de la física clásica como lo expresa (Ruiza, Fernández, y Tamaro, 2004).

Britannica (s.f) describe a Carl Friedrich Gauss nació en 1777 y falleció en 1855, es considerado como uno de los tres más grandes matemáticos de la historia de la matemática, revolucionó la aritmética, la astronomía y la estadística, descubrió la Campana de Gauss. Gauss sostenía que "la matemática es la reina de las ciencias, y la aritmética, la reina de las matemáticas". Su teorema muy conocido fue el teorema fundamental del álgebra, atribuyendo la expresión, toda expresión algebraica de grado mayor que cero tiene una raíz y en 1801 publicó su obra "Disquisitiones arithmeticae". Su pensamiento muy estudiantil "No es el conocimiento, sino el acto de aprendizaje, y no la posesión, sino el acto de llegar allí, que concede el mayor disfrute".

\section{Discusión}

Los docentes del Ministerio de Educación, las universidades, los establecimientos públicos y privados de nivel superior, medio y primario, deberán preocuparse por mejorar los resultados en las evaluaciones de matemáticas. Valorar y aplicar la reflexión filosófica sobre las matemáticas la cual está presente de manera explícita e implícita en los orígenes de la filosofía occidental. Tener en cuenta, la verdad como condición necesaria de la posibilidad del conocimiento, específicamente en la matemática en dónde hay que demostrarla. Ahora bien. ¿Es cognoscible todo aquello que es verdadero? Los filósofos, consideran que los objetos abstractos no existen de ninguna manera, ni independientemente de la mente del matemático ni como resultado de su actividad mental. Sin embargo, el idealismo establece, que los objetos matemáticos no tienen una existencia propia e independiente de la mente, sino que necesitan de la actividad de la 
mente para existir. Para los realistas, el universo matemático existe en toda su exuberancia con independencia de la mente humana, es natural suponer que hay verdades que están más allá de nuestras limitadas capacidades cognoscitivas.

Al reflexionar con los estudiantes la biografía de los filósofos y matemáticos Descartes, Arquímedes, Pascal, Newton y Gauss, se observó entusiasmo, esto permitió mejorar el aprendizaje de la matemática.

Por otra parte Villela-Cervantes (2018) afirma que la motivación es un factor que facilita el aprendizaje de la matemática, se lograrían mejores resultados en los aprendizajes si los docentes utilizaran metodologías lúdicas, como por ejemplo la metodología que utiliza el Maestro Adery Pérez la cual radica en determinar el costeo a través del juego, consiste en aplicar los números para resolver los problemas elementales de la vida diaria.

Por lo consiguiente, la educación marcó un antes y después de la pandemia provocada por el Coronavirus, el uso de herramientas tecnológicas virtuales permitió desarrollar las clases, en ese sentido es necesario que los docentes a través del aprendizaje autónomo conozcan las metodologías innovadoras que permitan el fácil acceso a los libros digitales, crear videos o utilizar los existentes en YouTube, para facilitar el aprendizaje de las matemáticas como ciencia compleja que necesita diversidad de herramientas para facilitar el aprendizaje (Villela-Cervantes, 2018).

\section{Agradecimientos}

Se agradece a la Escuela de Postgrado de la Facultad de Humanidades, a los profesores de la Maestría de Filosofía, en especial al maestro Juan Pablo Escobar, al asesor de tesis M.A Efraín Estrada por el acompañamiento en el caminar académico. 


\section{Referencias}

Britannica (s.f). Biographies. https://www.britannica.com/biographies.

Livio, M. (2009). ¿Es Dios un Matemático? Universidad del Valle, Cali, Colombia.

Mineduc. (2009). Resultados generales de la evaluación educativa. Guatemala. https://www.mineduc.gob.gt/digeduca/ documents/resultados/Resultados_generales.pdf

Ruiza, M., Fernández, T. y Tamaro, E. (2004). Biografia de Pitágoras. En Biografías y Vidas. La enciclopedia biográfica en línea. Barcelona (España). Recuperado de https://www. biografiasyvidas.com/biografia/p/pitagoras.htm el 2 de marzo de 2021.

Villela Cervantes, C. E. (2018). Metodología costeo por juegos. Revista Ciencia Multidisciplinaria CUNORI, 2(1), 115-116. https://doi. org/10.36314/cunori.v2i1.73

Villela Cervantes, C. E. (2018). La educación virtual en las ciencias complejas. Revista Académica Cunzac, 1(1), 1-6. https://doi. org/10.46780/cunzac.v1i1.1

Yarza de la Sierra, I. (2015). Philosophica: Enciclopedia filosófica on line. https://www.philosophica.info/voces/aristoteles/ Aristoteles.html 


\section{Sobre los autores}

\section{Rafael Rubén Sanic Maguirre}

Es ingeniero Industrial, graduado en la Maestría en Filosofía de la Universidad de San Carlos de Guatemala, se desempeño como profesor de matemáticas durante 32 años en el Colegio Italiano de Guatemala y también en el Instituto Tecnológico Universitario Guatemala Sur (ITUGS). Atendió los cursos Matemática Básica 1 y 2 durante ocho años y actualmente imparte los cursos Gestión Empresarial 1 y Ética Profesional. En la Universidad REGIONAL imparte el curso de Filosofía. Es autor del Manual Matemáticas financieras para estudiantes de bancos en INTECAP 2008.

\section{Claudia Esmeralda Villela Cervantes}

Es Doctorada en Educación, profesora investigadora, egresada de la Universidad Mariano Gálvez de Guatemala, labora como docente e investigadora en la Universidad de San Carlos de Guatemala.

\section{Copyright (c) Rafael Rubén Sanic Maguirre y Claudia Esmeralda Villela Cervantes}

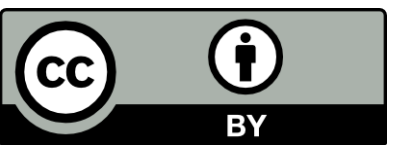

Este texto está protegido por una licencia Creative Commons 4.0.

Usted es libre para compartir, copiar y redistribuir el material en cualquier medio o formato y adaptar el documento, remezclar, transformar y crear a partir del material para cualquier propósito, incluso comercialmente, siempre que cumpla la condición de atribución: usted debe reconocer el crédito de una obra de manera adecuada, proporcionar un enlace a la licencia, e indicar si se han realizado cambios. Puede hacerlo en cualquier forma razonable, pero no de forma tal que sugiera que tiene el apoyo del licenciante o lo recibe por el uso que hace. 